\title{
II Sistema Nazionale Linee Guida (SNLG) e SIPMeL
}

\author{
Guidelines National System (SNGL) and SIPMeL (Italian Society of Clinical \\ Pathology and Laboratory Medicine)
}

\author{
Piero Cappelletti ${ }^{1}$ (I)
}

Ricevuto: 31 maggio 2018 / Accettato: 13 giugno 2018 / Pubblicato online: 22 giugno 2018

(C) Società Italiana di Patologia Clinica e Medicina di Laboratorio 2018

Riassunto Recentemente è stato presentato il Sistema Nazionale Linee Guida (SNLG) a cura dell'Istituto superiore di sanità (ISS), legato alla legge sulla responsabilità medica 24/2017. Sono operativi la piattaforma informatica SNGL per l'inserimento di linee guida, il Manuale metodologico per la produzione delle linee guida di pratica clinica e il Manuale operativo per la proposta di inserimento di linee guida nel Sistema Nazionale Linee Guida a cura di CNEC (Centro nazionale per l'eccellenza clinica, la qualità e la sicurezza delle cure) e gli standard internazionali di riferimento, costruiti con il concorso essenziale di GIMBE. Il sito SNLG di ISS presenta oggi le sezioni Editoriale, Informazioni, Buone Pratiche, Linee Guida (Consultazione, Valutazione e pubblicazione, Produzione) e Piattaforma SNLG. Così come concepito, il SNLG è lo strumento di riferimento medico-legale di linee guida e raccomandazioni per la pratica clinica e assistenziale, rivolta a grandi e selezionati temi clinici della professione, i cui attori sono un gruppo di enti a tradizione storica (Regioni; Istituti privati di ricerca e studio) e, quando sarà approvato l'elenco ex DM 2 agosto 2017, le Società scientifiche e le Associazioni tecnico-scientifiche in gruppi multidisciplinari e multiprofessionali, con prevalenza delle Società cliniche. Il ruolo della Medicina di Laboratorio potrà essere quello della necessaria partecipazione ai gruppi multidisciplinari e multiprofessionali per garantire conoscenze e competenze specifiche e la pubblicazione delle raccomandazioni di guidance per la buona pratica di laboratorio. L'obbligatorietà del primo punto e una specifica area per il secondo dovranno essere formalmente richieste all'ISS.

Parole chiave SNLG $\cdot$ Linee guida $\cdot$ LMPG

$凶$ P. Cappelletti

pie.cappelletti@gmail.com

1 SIPMeL, Castelfranco Veneto, TV, Italia
Summary Recently, a Guidelines National System (SNGL) was founded in Italy as the point of elaboration of national guidelines (GLs) for medico-legal issues, following the law 24/2017. Now, the Methodological Manual, the Operative Manual, the informatics platform and the adopted standards are operative. The site is dedicated to Clinical Practice Guidelines $(C P G)$ of relevant scope and interest, proposed by authorized public and private Institutes and Scientific Societies with certified characteristics. The role of Laboratory Medicine and SIPMeL should be both: participating to multidisciplinary and multiprofessional groups and collaborating with clinical Scientific Societies for consensus, and defining guidance for "internal" issues in the production of the laboratory information to the clinic and preparing specific recommendations ( $L M P G$ ) for the steps of total testing process, ignored by general CPG, to answer adequately to the clinical question. SIPMeL should ask ISS (Istituto superiore di sanità), that manages $S N G L$, for a formal definition of the first point in the requested procedures for GLs elaboration and for a specific area of website as repository of LMPGs.

Keywords SNGL $\cdot$ Guidelines $\cdot L M P G$

\section{Linee guida e responsabilità medica}

Secondo la presentazione del nuovo Sistema Nazionale Linee Guida (SNLG) dell'Istituto superiore di sanità (ISS) (https://snlg.iss.it), "le linee guida di pratica clinica sono uno strumento di supporto decisionale finalizzato a consentire che, tra opzioni alternative, sia adottata quella che offre un migliore bilancio tra benefici ed effetti indesiderati, tenendo conto dell'esplicita e sistematica valutazione delle prove disponibili, commisurandola alle circostanze peculiari del 
caso concreto e condividendola-laddove possibile—con il paziente o i caregivers" [1].

Il SNLG è conseguenza della Legge 24 dell'8 marzo 2017 sulla responsabilità medica che stabilisce, all'art. 5, che gli esercenti le professioni sanitarie nell'esecuzione delle prestazioni sanitarie debbano attenersi, salve le specificità del caso concreto, alle raccomandazioni previste dalle linee guida elaborate da enti e istituzioni pubbliche e private e da Società scientifiche e Associazioni tecnico-scientifiche delle professioni sanitarie iscritte in apposito elenco e pubblicate dall'ISS nel SNLG, previa verifica di conformità della metodologia adottata a standard internazionalmente riconosciuti e resi pubblici da parte dell'Istituto stesso, e della rilevanza delle evidenze scientifiche dichiarate a supporto delle raccomandazioni.

Il primo passo attuativo della 24/2017 è stato la predisposizione dell'elenco delle Società scientifiche e Associazioni tecnico-scientifiche accreditate a tal fine, istituito e regolamentato con DM 2 agosto 2017, che prevede alcuni requisiti: rilevanza nazionale e regionale, rappresentatività del 30\% dei professionisti per disciplina (15\% per medici di medicina generale), statuto con espressi contenuti come denominazione, sede, patrimonio, autonomia e indipendenza, assenza di ruolo scientifico, democraticità dell'elezione degli organi, ruolo disciplinare, accessibilità, assenza di finalità di lucro, pubblicazione sul sito web dell'attività scientifica, regolamentazione del conflitto d'interesse, ruolo del Comitato scientifico e altre specifiche di garanzia. La definitiva stesura è bloccata dall'interpretazione del requisito della rappresentatività (peraltro di assai dubbia scientificità), che forse sarà riferita alle strutture piuttosto che ai singoli professionisti.

\section{Il sistema nazionale linee guida}

Il secondo passo è stato il DM 27 febbraio 2018 che prevede l'istituzione di un "nuovo" SNLG-nuovo perché già nel 1998 e nel 2006 il SSN aveva previsto un Piano Sanitario Linee Guida-PNLG che ebbe scarsa fortuna-gestito dall'ISS tramite il CNEC (Centro nazionale per l'eccellenza clinica, la qualità e la sicurezza delle cure) e la definizione delle procedure per l'elaborazione e l'approvazione delle linee guida. Per elaborazione di linee guida si intende la produzione $e x$ novo, l'adattamento nazionale di linee guida internazionali e l'aggiornamento di linee guida precedenti (tempo di obsolescenza $>3$ anni). SNLG è l'unico punto d'accesso per linee guida previste dall'art. 5 della 24/2017, cioè a fini medicolegali. La gestione è affidata a un Comitato strategico costituito dal presidente dell'ISS, in qualità di coordinatore; dal direttore del CNEC dell'ISS; dal direttore generale della programmazione sanitaria del Ministero della salute; dal direttore generale della prevenzione sanitaria del Ministero della salute; dal direttore generale delle professioni sanitarie e delle risorse umane del Servizio sanitario nazionale del
Ministero della salute; dal direttore generale della ricerca e dell'innovazione in sanità; dal direttore generale dell'Agenzia per i servizi sanitari regionali (AGENAS); dal direttore generale dell'Agenzia italiana del farmaco (AIFA); dal presidente del Consiglio superiore di sanità; da quattro rappresentanti della Commissione salute della Conferenza delle regioni e delle province autonome. Ha le funzioni di definire le priorità, garantire l'efficienza del sistema, monitorare l'attività, relazionare al Ministero. Le priorità vengono definite sulla base dei seguenti criteri: impatto epidemiologico delle malattie sulla popolazione italiana; variabilità delle pratiche professionali non giustificate dalle evidenze disponibili; diseguaglianze di processi ed esiti assistenziali; benefici potenziali derivanti dalla produzione di linee guida; tipo e qualità delle evidenze disponibili; rischio clinico elevato; istanze sociali e bisogni percepiti dalla popolazione. Può avvalersi di collaborazioni esterne cliniche, scientifiche, di ricerca e di associazioni di pazienti. L'art. 4 del DM prevede che entro 30 giorni l'ISS determini gli standard metodologici e i criteri di valutazione delle linee guida per l'inserimento delle stesse nel SNLG e l'art. 5 dettaglia l'inserimento delle linee guida in SNLG: piattaforma informatica ove inserire le linee guida (titolo, argomento, contenuti, destinatari); attori (enti pubblici e privati e Società Scientifiche presenti nell'apposito elenco); caratteristiche dell'inserimento (ex novo, adattamento, aggiornamento di $\mathrm{LG}$ ); valutazione di ammissibilità da parte di ISS secondo alcuni criteri (priorità stabilite dal Comitato strategico; eventuale disponibilità di linee guida aggiornate, nazionali o internazionali, adottabili nel contesto italiano; copertura dell' area clinica di interesse, da parte di linee guida già inserite nel SNLG); tempi di reazione del sistema (ammissibilità 30 giorni; comunicazione al Comitato Strategico delle linee guida ammesse in 15 giorni) e della preparazione della redazione definitiva delle linee guida per il giudizio di merito (non meno di 6 mesi e non più di 2 anni dalla data dell'ammissione; ricerca bibliografica entro gli ultimi 12 mesi).

\section{Gli strumenti del SNLG}

Il 7 maggio è stata presentata la piattaforma informatica SNGL (https://piattaformasnlg.iss.it), gestita da ISS, il Manuale metodologico per la produzione delle linee guida di pratica clinica (https://snlg.iss.it/wp-content/uploads/2018/ 06/MM_v1.1_mag-2018.pdf) a cura di CNEC, il Мапиаle operativo per la proposta di inserimento di linee guida nel Sistema Nazionale Linee Guida (https://snlg.iss.it/wpcontent/uploads/2018/04/manuale-operativo-SNLG-v2_ aprile-2018.pdf) sempre a cura di CNEC e gli standard internazionali di riferimento, costruiti con il concorso essenziale di GIMBE. Il sito SNLG di ISS (https://snlg.iss.it) presenta oggi le sezioni Editoriale, Informazioni, Buone Pratiche, 
Linee Guida (Consultazione, Valutazione e pubblicazione, Produzione) e Piattaforma SNLG.

Il Manuale metodologico [2] contiene nell'introduzione le informazioni relative ai concetti di linee guida, alle finalità e destinatari del manuale, ai criteri di prioritarizzazione; specifica i gruppi coinvolti nella produzione di linee guida (comitato tecnico-scientifico, quality assurance team, panel di esperti, gruppo di lavoro, referee esterni, stakeholder) e il tema del conflitto di interessi; esamina lo sviluppo dello scope e il percorso dal quesito clinico alle raccomandazioni con attenzione all'individuazione degli outcome, alla ricerca delle evidenze e loro valutazione; suggerisce schemi di PDTA e indicatori di audit, come validare la bozza di LG e rispondere agli stakeholder e revisionare esternamente la linea guida; indica come finalizzare, pubblicare e disseminare le linee guida e i tempi e modi dell'aggiornamento.

I concetti portanti sono la composizione multiprofessionale e multidisciplinare dei proponenti, dei panel e dei gruppi operativi; la scelta del metodo GRADE come framework metodologico raccomandato dal CNEC per la produzione di linee guida destinate al SNLG; l'indicazione a previlegiare gli adattamenti nazionali di linee guida internazionali con il metodo GRADE-ADOLOPMENT; la scelta del metodo AGREE II per la valutazione metodologica della qualità delle LG.

Gli standard internazionali esaminati per la Qualità delle linee guida sono G-I-N (Guidelines International Network) [3], prevalentemente destinato ai produttori di linee guida ex novo, IOM (Institute of Medicine) [4] e AGREE II (Appraisal of Guidelines for Research \& Evaluation) [5], prevalentemente destinato ai valutatori, e lo strumento semplificato AGREE Global Scale Rating [6], recentemente proposto per esperti AGREE. Il confronto tra i diversi metodi premia AGREE II per la maggior completezza specialmente in riferimento all'item Applicabilità. La Completezza delle linee guida è garantita dall'uso di strumenti come il RIGHT (Reporting Items for practice Guidelines in Healthcare) Statement [7] o, ancora meglio, da AGREE Reporting Checklist [8]. Per l'Adattamento, gli strumenti internazionali sono il G-I-N Adaptation Working Group [9], che ha sostituito dal 2010 l'ADAPT Collaboration, e il GRADEADOLOPMENT [10], crasi di adoption e devolopment, all'interno del GIMBE framework per l'applicabilità che prevede requisiti minimi di accreditamento (STOP: Strutturali, Tecnologici, Operativi, Professionali), criteri di normativa sanitaria (LEA), fattori socio-culturali, orografia e viabilità. Per l'Aggiornamento, il riferimento è la CheckUp Checklist [11], recentemente pubblicata e un tempo di revisione SNLG di 3 anni. Per il Conflitto di Interessi (principi e gestione di conflitti finanziari e non-finanziari) e la disclosure dello stesso ci si rifà a G-I-N [9].

Il Manuale operativo [12] per l'inserimento di linee guida nella piattaforma SNLG poggia sugli standard AGREE

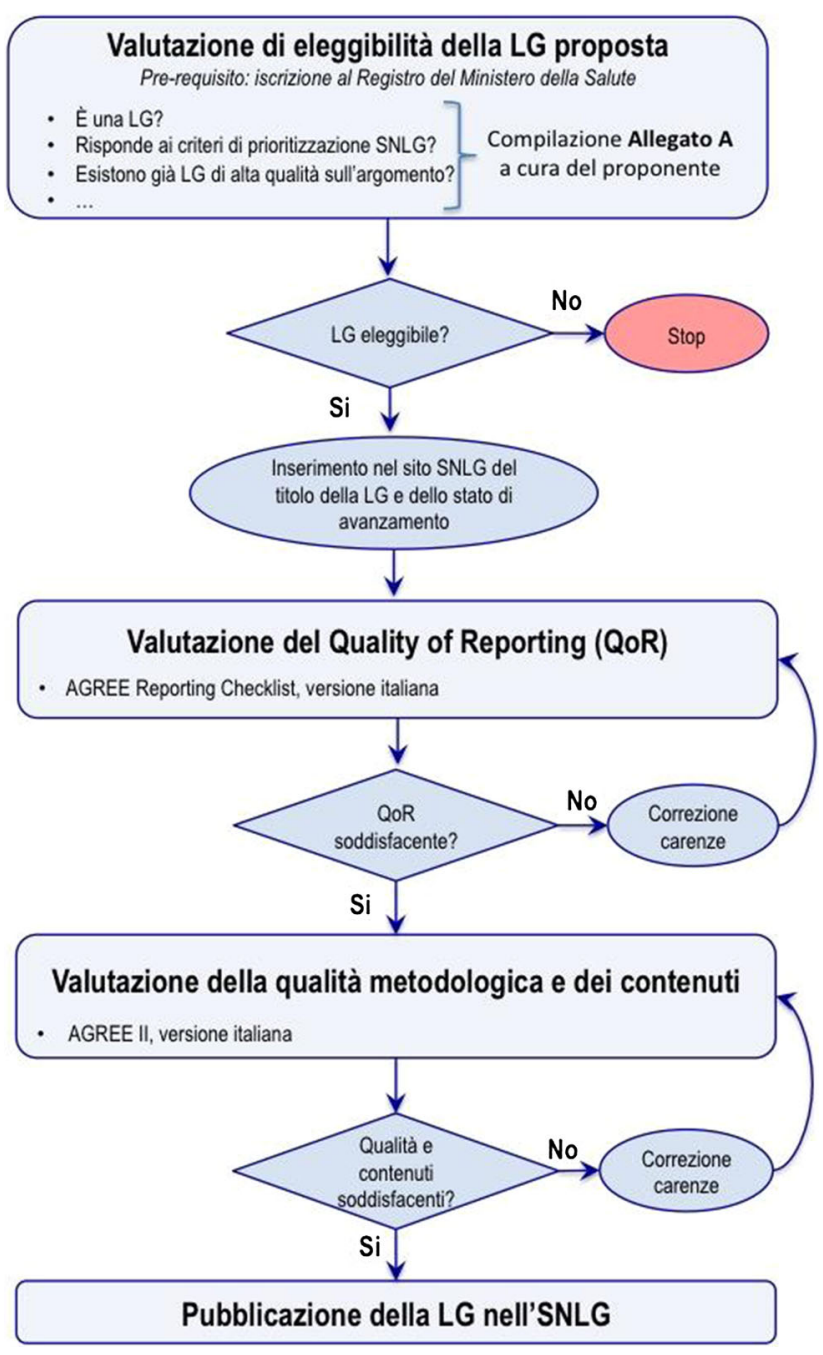

Fig. 1 Workflow per l'inserimento di linee guida (LG) nella piattaforma SNLG. Tratta dal Manuale operativo per la proposta di inserimento di linee guida nel Sistema Nazionale Linee Guida (https://snlg.iss.it/wp-content/uploads/2018/04/manuale-operativoSNLG-v2_aprile-2018.pdf)

Reporting Checklist e AGREE II versione italiana a cura di GIMBE. Il workflow è sintetizzato nella Figura 1 e contempla un primo passaggio di eleggibilità, composto dalla verifica del soggetto proponente (ente autorizzato o Società scientifica nell'elenco apposito), dell'esistenza o meno di altre linee guida con lo stesso scope e dell'aderenza alle priorità stabilite (Allegato A); poi un controllo di completezza; quindi la valutazione della qualità (QoR) sotto il profilo metodologico e nel merito, da cui possono derivare la necessità e il percorso di correzioni; infine l'iter di pubblicazione (il proponente non la pubblica prima della comparsa in SNLG, la ritira se emergono evidenze nuove, accetta commenti in rete, ha tempi prestabiliti per i vari passaggi ecc.). A oggi, l'ISS con Decreto 13/2018 ha individuato 48 soggetti collaboratori autorizzati all'inserimento di linee guida, mentre l'elenco delle Società scientifiche e Associazioni tecnico-scientifiche 
non è pubblicato e quindi solo i primi soggetti sono autorizzati a inserire proposte di linee guida. Le linee guida in consultazione (approvate) sono 1; quelle in valutazione 14 .

L'insieme delle procedure e dei contenuti di SNGL è certamente di elevato spessore contenutistico e metodologico. I rischi del sistema sono ben presenti anche ai costruttori [1]: considerati i tempi medi di produzione di una linea guida (12-24 mesi) o anche di un suo adattamento o revisione, "è irrealistico che SNGL possa essere rapidamente popolato da un numero di linee guida commisurato alle esigenze", teoricamente infinite come i problemi della pratica medica, ed esiste il pericolo che esso "contenga cristallizzazioni di raccomandazioni in perenne ritardo rispetto allo sviluppo, talora tumultuoso, della ricerca medica". Soprattutto le LG non devono essere intese come "scorciatoie mentali per decisioni stereotipate".

Quando non vi sono linee guida approvate, SNLG offre sul sito https://snlg.iss.it il box Buone Pratiche, che contiene le buone pratiche clinico-assistenziali già presenti nella vecchia versione dell'SNLG, purché con data di pubblicazione non superiore a 3 anni, e le buone pratiche identificate dal CNEC attraverso un processo di ricognizione della letteratura biomedica, la sorveglianza attiva delle principali banche dati di linee guida e Revisioni sistematiche, dei documenti pubblicati dalle agenzie nazionali e internazionali che producono linee guida, e delle best practices riconosciute con meccanismi di consenso tra esperti, a livello nazionale e internazionale. I criteri adottati per la selezione delle buone pratiche sono: rilevanza dell'argomento; data di pubblicazione $<3$ anni; composizione multidisciplinare e multiprofessionale del panel di esperti. Esso contiene le linee guida Regionali, documenti GREFO (Gruppo Regionale Farmaci Oncologici Emilia-Romagna), documenti AGENAS, le sintesi italiane delle linee guida NICE e Choosing Wisely edizione italiana.

\section{SNLG e medicina di laboratorio}

Così come concepito, il SNLG è lo strumento di riferimento medico-legale di linee guida e raccomandazioni per la pratica clinica ed assistenziale, rivolta a grandi e selezionati temi clinici della professione; gli attori sono un gruppo di enti a tradizione storica (Regioni; Istituti privati di ricerca e studio) e, quando sarà approvato l'elenco ex DM 2 agosto 2017, le Società scientifiche e le Associazioni tecnico-scientifiche in gruppi multidisciplinari e multiprofessionali, con prevalenza delle Società cliniche; lo spazio per la Medicina di Laboratorio è oggettivamente limitato, anche se l'impostazione data da SIPMeL al tema delle linee guida esce rafforzata dal confronto con SNLG, in particolare rispetto agli standard metodologici di riferimento.

SIPMeL, infatti, ha ridefinito la propria politica rispetto alle linee guida nel 2015 [13], sottolineando le specificità delle Linee Guida per la Pratica di Laboratorio (LMPG) e dei loro riferimenti internazionali (IOM, NACB) e le differenze metodologiche (scarsa efficienza del metodo GRADE) e contenutistiche (prevalenza di raccomandazioni di guidance piuttosto che di comportamento clinico) rispetto alle linee guida cliniche e assistenziali. Tali temi, insieme con le procedure per la produzione, adattamento e aggiornamento di LMPG, sono stati affrontati in un workshop tenutosi in occasione del Consiglio Nazionale del 18 maggio 2017 e i materiali e il documento da esso derivati sono stati messi a disposizione sul sito della Società per osservazioni e integrazioni. Il documento finale è stato portato all'approvazione del Consiglio Nazionale (CN) di Riva del Garda il 9 ottobre 2017 per diventare norma della Società. Di conseguenza è stata operata una revisione delle LMPG prodotte negli ultimi 7 anni e approvate dalla Società e, a norma di Statuto, sono state pubblicate in un'area del sito societario (https://www.sipmel.it/it/) denominata Linee Guida e organizzata in Metodologia per Linee Guida SIPMeL, dove sono visibili i principi, i criteri, i percorsi e le finalità di SIPMeL riguardo le linee guida; Linee Guida SIPMeL approvate da $\mathrm{CN}$, dove sono rinvenibili le linee guida che sono state formalmente approvate dal Consiglio Nazionale e sono patrimonio della Società; Linee Guida SIPMeL in sviluppo o in revisione, dove sono visibili i draft e i position paper aperti alla discussione in vista della prima approvazione oppure della verifica delle revisioni ed aggiornamenti; Linee Guida sostenute da SIPMeL, dove sono raccolte le linee guida che non sono state prodotte o adattate da SIPMeL ma che la Società reputa di riferimento anche per la buona pratica di Laboratorio. Attualmente, vi sono 25 linee guida approvate dal $\mathrm{CN}$ che quando affrontano temi prettamente clinici si rifanno solo al tema della selezione dei test; 9 nel box sviluppo/revisione principalmente perché di antica elaborazione; 2 stanno per essere inserite nell'ultimo gruppo delle linee guida sostenute da SIPMeL [14].

Le possibilità di partecipazione di SIPMeL a SNLG sono sostanzialmente due.

Da un lato vi è la partecipazione della Società e dei suoi esperti al gruppo di proponenti e ai gruppi operativi di linee guida cliniche insieme con Società cliniche, in particolare quando la diagnostica di laboratorio sia uno strumento indispensabile o critico per la diagnosi e il monitoraggio della malattia e terapie del problema clinico in questione. La ratio sta nella dimostrata importanza della presenza di un patologo clinico nella definizione accurata delle caratteristiche operative e diagnostiche dei test in questione. Vi è un'enorme quantità di linee guida per la pratica clinica, di buon livello, preparate da produttori internazionali, talché molto spesso gli argomenti sono già stati affrontati in modo clinicamente adeguato. Tuttavia è noto [15] come le linee guida cliniche abbiano generalmente una bassa considerazione per gli aspetti considerati laboratoristici, rispettando in media 
solo in un terzo dei casi le necessarie informazioni riguardo lo stato dei pazienti, le interferenze biologiche e analitiche e il trattamento dei campioni, e che l'inserimento di uno specialista di laboratorio nei comitati/gruppi di preparazione di line guida cliniche migliora la situazione rendendone circa la metà esaustive sotto il profilo dei mezzi diagnostici. Vi è quindi la necessità che SNLG metta in evidenza, nelle procedure e nella prassi, l'obbligatorietà della presenza della Medicina di Laboratorio e della sua Società Scientifica nei gruppi di elaborazione di linee guida dove la diagnostica di laboratorio sia importante. Questa deve essere una richiesta specifica che SIPMeL deve rivolgere a ISS, con il supporto di FISMELab e di FISM.

In secondo luogo, come già ricordato, il compito principale di SIPMeL è rivolto alla definizione o adattamento di LMPG per la guidance della buona pratica, utilizzando in maniera sistematica e ragionata quanto disponibile per fornire raccomandazioni utili ai professionisti della Medicina di Laboratorio a risolvere concreti problemi giornalieri che consentono risultati e risposte efficaci nel percorso clinico del paziente. SIPMeL raccomanda di prestare attenzione a tutte le fasi del Total Testing Process e, in particolare, di quelle fasi e di quei passaggi (fase preanalitica diversa dalla richiesta dell'esame; fase analitica; fase postanalitica interna al Laboratorio) trascurati dalle linee guida cliniche che si occupano prevalentemente, dal punto di vista diagnostico, di appropriatezza della richiesta di test diagnostici e del loro ruolo nel percorso diagnostico, di rule in/rule out e di follow-up. Nella pratica del laboratorio, invece, sono spesso gli aspetti tecnologici e organizzativi che hanno la necessità di essere indirizzati, soprattutto quando questi aspetti che possono apparire "interni" rispetto all'interfaccia clinica-laboratorio condizionano invece pesantemente il percorso diagnostico e quindi terapeutico del paziente [15]. Le LMPG, oggi, non potrebbero trovare spazio nel box Buone Pratiche del sito SNLG, anch'esse orientate totalmente alla pratica clinica. È necessario, pertanto, richiedere a ISS l'individuazione di un'area del sito SNLG in cui collocare le LMPG. Tale richiesta dovrà trovare il supporto di FISMELab e FISM, anche perché risolve un tema presente a tutte le discipline della Medicina di Laboratorio e alle Società di diagnostica medica.
Con queste integrazioni il sito SNLG potrà diventare un riferimento non solo per gli aspetti medico-legali ma anche scientifico-pratici per tutte le discipline della Medicina.

Conflitti di interesse Nessuno.

Studi condotti su esseri umani e animali Per questo tipo di studio non è richiesto l'inserimento di alcuna dichiarazione relativa agli studi effettuati su esseri umani e animali.

\section{Bibliografia}

1. https://snlg.iss.it (Accesso 19 maggio 2018)

2. https://snlg.iss.it/wp-content/uploads/2018/06/MM_v1.1_mag2018.pdf (Accesso 19 maggio 2018)

3. Qaseem A, Forland F, Macbeth F et al (2012) Guidelines international network: toward international standards for clinical practice guidelines. Ann Intern Med 156:525-531

4. Institute of Medicine (2011) Clinical practice guidelines we can trust. The National Accademies Press, Washington, DC

5. Brouwers MC, Kho ME, Browman GP et al (2010) AGREE II: advancing guideline development, reporting and evaluation in health care. CMAJ 182:e839-e842

6. Brouwers MC, Kho ME, Browman GP et al (2012) The global rating scale complements the AGREE II in advancing the quality of practice guidelines. J Clin Epidemiol 65:526-534

7. Chen Y, Yang K, Marušić A et al (2017) A reporting tool for practice guidelines in health care: the RIGHT statement. Ann Intern Med 166:128-132

8. Brouwers MC, Kerkvliet K, Spithoff K (2016) The AGREE reporting checklist: a tool to improve reporting of clinical practice guidelines. BMJ 352:i1152

9. http://www.g-i-n.net/working-groups/adaptation (Accesso 19 maggio 2018)

10. Schünemann HJ, Wiercioch W, Brozek J et al (2017) GRADE Evidence to Decision (EtD) frameworks for adoption, adaptation, and de novo development of trustworthy recommendations: GRADE-ADOLOPMENT. J Clin Epidemiol 81:101-110

11. Vernooij RW, Alonso-Coello P, Brouwers M et al (2017) Reporting items for updated clinical guidelines: checklist for the reporting of updated guidelines (check up). PLoS Med 14:e1002207

12. https://snlg.iss.it/wp-content/uploads/2018/04/manuale-operativoSNLG-v2_aprile-2018.pdf (Accesso 19 maggio 2018)

13. Cappelletti P (2015) Linee guida e SIPMeL. Riv Ital Med Lab $11: 185-190$

14. https://www.sipmel.it/it/ (Accesso 19 maggio 2018)

15. Aakre KM, Langlois MR, Watine J et al (2013) Critical review of laboratory investigations in clinical practice guidelines: proposals for the description of investigation. Clin Chem Lab Med 51:12171226 